\title{
Exponential stability in thermoelasticity with microtemperatures
}

\author{
Pablo S. Casas \\ Dept. Matemática Aplicada 1, U.P.C., \\ Diagonal, 647. 08028 Barcelona, Spain \\ e-mail: pablo@casas.upc.es -Web page: http://www-ma1.upc.es/^casas \\ Ramón Quintanilla \\ Dept. Matemática Aplicada 2, U.P.C., \\ Colom, 11. 08222 Terrassa, Barcelona, Spain
}

keywords: thermoelasticity with microtemperatures, exponential stability.

Running head: Exponential stability in thermoelasticity.

\begin{abstract}
This article is concerned with a linear theory for elastic materials with inner structure, whose particles in addition to the classical displacement, possess microtemperatures. In the main part of the paper we restrict our attention to the one-dimensional problem. First, we prove the slow decay of solutions for the onedimensional problem of micromorphic elastic solids with the usual thermal effects. Then, we prove the exponential stability of the solutions when we consider the theory with microtemperatures. The anti-plane distributions of microtemperatures are considered later.
\end{abstract}

Acknowledgment: This work is part of the project "Stability aspects in thermomechanics" (BFM2003-00309) of the Spanish Ministry of Science and Technology. The second author (R.Q.) wants to thank to an anonymous referee the comments concerning the slow decay on a previous manuscript related with these questions. 
Exponential stability in thermoelasticity with microtemperatures

\section{Introduction}

Much has been written on the subject of the theory of elastic solids in which the deformation is described not only by the usual vector displacement field, but by other vector or tensor fields as well. It is not our intention to review the vast literature, but we can recall some classical references in this topic $[1,4,5,6,7]$. In the continuum theories with microstructure the microelements undergo homogeneous deformations called microdeformations. On the basis of Eringen's theory of micromorphic continua, Grot [3] developed a theory of thermodynamics for elastic materials with microstructure whose microelements, in addition to microdeformations, possess microtemperatures. The Clausius-Duhem inequality is modified to include microtemperatures, and the first-order moment of the energy equations are added to the usual laws of a micromorphic continuum. The theory of micromorphic fluids with microtemperature has been studied in various papers (see $[18,20])$. It is also worth recalling some contributions to thermoelastic solids with microstructure and/or microtemperature [9, 10, 11, 12, 13] A study of heat conduction in materials with inner structure was presented by Riha [19]. Experimental data for the silicone rubber containing spherical aluminium particles and for the human blood were found to conform closely to predict theoretical thermal conductivity.

Most of the works devoted to the theory of thermoelastic solids with microstructure assume that each microelement is capable of undergoing an affine deformation and has uniform temperature. In this article we consider a linear theory for elastic solids with inner structure whose particles, in addition to the classical displacement, microstretch and thermal fields, possess microtemperatures. This is one of the simplest thermomechanical theory of elastic solids that takes into account the new thermal variables. We restrict our attention to the one-dimensional theory and we prove several results of exponential stability for the solutions of the thermoelasticity with microtemperatures.

In recent years a relevant task has been developed to obtain exponential stability of solutions in thermoelastic theories. The classical theory was first considered by Dafermos [2] and Slemrod [22] and it has been studied in the book of Jiang and Racke [14] and the contribution of Lebeau and Zuazua [15]. The Lord and Shulmann theory of thermoelasticity has been studied by Racke [21] and the thermoelasticity of type III has been considered by Quintanilla and Racke [17] and Zhang and Zuazua [24]

The paper is structured as follows: In section two we recall the basic equations which govern the problem of thermo-microstretch elastic solids with microtemperatures. The one-dimensional problem is considered. In section three we see that generically, solutions of the problem of thermo-microstretch elastic solids decay slowly. The exponential decay of solutions when microtemperatures are considered is proved in section four. In section five we restrict our attention to the heat conduction problem in three dimensions and we point out the existence of anti-plane distributions of microtemperatures. 


\section{Basic Equations}

We consider the linear theory of thermodynamics for isotropic elastic materials with inner structure.

The equations which govern the thermoelastic deformations of microstretch materials with microtemperatures are (see [9]) :

$$
\begin{gathered}
\mu \triangle \mathbf{u}+(\lambda+\mu) \operatorname{grad} \operatorname{div} \mathbf{u}+b \operatorname{grad} \phi-\beta \operatorname{grad} \theta=\rho \ddot{\mathbf{u}} \\
\alpha \triangle \phi-b \operatorname{div} \mathbf{u}-\xi \phi-d \operatorname{div} \mathbf{w}+m \theta=J \ddot{\phi} \\
k \triangle \theta-\beta T_{0} \operatorname{div} \dot{\mathbf{u}}+\kappa_{1} \operatorname{div} \mathbf{w}-m T_{0} \dot{\phi}=c \dot{\theta} \\
\kappa_{6} \triangle \mathbf{w}+\left(\kappa_{4}+\kappa_{5}\right) \operatorname{grad} \operatorname{div} \mathbf{w}-d \operatorname{grad} \dot{\phi}-\kappa_{3} \operatorname{grad} \theta-\kappa_{2} \mathbf{w}=\delta \dot{\mathbf{w}}
\end{gathered}
$$

where $c=a T_{0}$. Here $\rho$ is the reference mass density, $\mathbf{u}=\left(u_{i}\right)$ is the displacement vector; $\theta$ is the temperature measured from the constant absolute temperature $\left(T_{0}>0\right)$; $\lambda, \mu, \beta, a, b, k, \xi, J, m, d, \alpha, \kappa_{i}(i=1, \ldots 6)$ are constitutive coefficients; $\mathbf{w}=\left(w_{i}\right)$ is the microtemperature vector and $\phi$ is the microstretch. We have used a superposed dot to denote partial differentiation with respect to time.

The Clausius-Duhem inequality implies that

$$
\begin{gathered}
3 \kappa_{4}+\kappa_{5}+\kappa_{6} \geq 0, \quad \kappa_{5}+\kappa_{6} \geq 0, \\
\kappa_{6}-\kappa_{5} \geq 0, \quad k \geq 0, \quad\left(\kappa_{1}+T_{0} \kappa_{3}\right)^{2} \leq 4 T_{0} k \kappa_{2} .
\end{gathered}
$$

Although in section five we consider the three-dimensional problem for the heat conduction, the main aim of this paper concerns the thermomechanical one-dimensional problem. Thus equations (2.1)-(2.4) reduce to

$$
\begin{gathered}
\mu^{*} u_{x x}+b \phi_{x}-\beta \theta_{x}=\rho \ddot{u} \\
\alpha \phi_{x x}-b u_{x}-\xi \phi-d w_{x}+m \theta=J \ddot{\phi} \\
k \theta_{x x}-\gamma \dot{u}_{x}-l \dot{\phi}+\kappa_{1} w_{x}=c \dot{\theta} \\
\kappa_{4}^{*} w_{x x}-d \dot{\phi}_{x}-\kappa_{3} \theta_{x}-\kappa_{2} w=\delta \dot{w}
\end{gathered}
$$

where $\mu^{*}=\lambda+2 \mu, \kappa_{4}^{*}=\kappa_{4}+\kappa_{5}+\kappa_{6}, \gamma=T_{0} \beta, l=T_{0} m$. It is worth noting that the couples $\beta, \gamma$ and $l, m$ have the same sign. To save cumbersome notation we will omit the stars in the notation of $\mu^{*}$ and $\kappa_{4}^{*}$.

In order to have a well posed problem we need to impose the initial conditions

$$
\begin{gathered}
u(x, 0)=u^{0}(x), \quad \dot{u}(x, 0)=v^{0}(x), \quad \phi(x, 0)=\phi^{0}(x), \quad \dot{\phi}(x, 0)=\varphi^{0}(x), \\
\theta(x, 0)=\theta^{0}(x), \quad w(x, 0)=w^{0}(x), x \in(0, \pi)
\end{gathered}
$$

where $u^{0}, v^{0}, \phi^{0}, \varphi^{0}, \theta^{0}$ and $w^{0}$ are given, and the boundary conditions

$$
\begin{gathered}
u=0\left(\text { or } u_{x}=0\right), \quad \phi=0\left(\text { or } \phi_{x}=0\right), \\
\theta=0\left(\text { or } \theta_{x}=0\right), \quad w=0\left(\text { or } w_{x}=0\right), \text { for } x=0, \pi
\end{gathered}
$$

From now on, we assume that the mechanical constitutive constants satisfy

$$
\rho>0, J>0, \mu>0, \alpha>0, \xi>0, \mu \xi>b^{2} .
$$


We also assume that $c$ and $\delta$ are positive.

It is worth noting that when the microtemperatures are not taken into account our system reduces to

$$
\begin{gathered}
\mu u_{x x}+b \phi_{x}-\beta \theta_{x}=\rho \ddot{u} \\
\alpha \phi_{x x}-b u_{x}-\xi \phi+m \theta=J \ddot{\phi} \\
k^{*} \theta_{x x}-\beta \dot{u}_{x}-m \dot{\phi}=a \dot{\theta}
\end{gathered}
$$

Here $k^{*}=T_{0}^{-1} k$, but we will drop the star in the next section. Thus, we can write $(2.16)$ as

$$
k \theta_{x x}-\beta \dot{u}_{x}-m \dot{\phi}=a \dot{\theta}
$$

In this paper we obtain several results on the stability for solutions of system (2.7)(2.10) with initial and boundary conditions (2.11), (2.12).

\section{Slow decay}

In this section we consider the problem determined by the system (2.14)-(2.16). We prove that generically the decay is slow. To this end we give two proofs. The first one is slower and only works for a particular class of boundary conditions. The second one is faster and more general. The main advantage of the first one is that the proof is more elemental and we do not need the use of functional analysis: elementary spectral arguments are enough, but the process is full of cumbersome calculations. These can be saved with the help of a program for algebraic manipulations. The second method needs arguments concerning functional analysis, and has the disadvantage that it only works when $\rho=J=a$.

In the first approach we restrict our attention to the boundary conditions

$$
u(x, t)=\phi_{x}(x, t)=\theta_{x}(x, t)=0, \quad x=0, \pi
$$

Whenever boundary conditions (3.1) are assumed we impose that

$$
\int_{0}^{\pi} \varphi^{0}(x) d x=\int_{0}^{\pi} \phi^{0}(x) d x=\int_{0}^{\pi} \theta^{0}(x) d x=0 .
$$

Our aim is to prove that for all positive $\epsilon$ sufficiently small, there exists a solution of the form

$$
u=A \exp (\omega t) \sin n x, \quad \phi=B \exp (\omega t) \cos n x, \quad \theta=C \exp (\omega t) \cos n x .
$$

such that $\operatorname{Re}(\omega)>-\epsilon$. In these conditions, we can always find a solution $\omega$ as near as we want of the imaginary axis. This fact implies that we can not obtain uniform exponential decay for solutions of the problem determined by system (2.14)-(2.16).

The solutions in this case will be combinations of functions of the form (3.3). Imposing (3.3) as a solution of the equations (2.14)-(2.16) we obtain the following homogeneous 
system with unknowns $A, B, C$ :

$$
\begin{aligned}
A \rho \omega^{2} & =-A \mu n^{2}-B b n+\beta n C, \\
B J \omega^{2} & =-B \alpha n^{2}-A b n-B \xi+m C, \\
C a \omega & =-k C n^{2}-A \beta n \omega-m B \omega,
\end{aligned}
$$

Our aim is to obtain a nontrivial solution of this linear system. We impose that its determinant vanishes. Therefore $\omega$ must be a solution of the equation

$$
\begin{aligned}
& \rho x^{5} J a+\rho x^{4} J k n^{2}+\left(\mu n^{2} J a+\beta^{2} n^{2} J+\rho \alpha n^{2} a+\rho \xi a+\rho m^{2}\right) x^{3}+ \\
& \left(\mu n^{4} J k+\rho \alpha n^{4} k+\rho \xi k n^{2}\right) x^{2}+\left(-2 b n^{2} m \beta+\mu n^{2} m^{2}+\beta^{2} n^{4} \alpha-\right. \\
& \left.b^{2} n^{2} a+\mu n^{2} \xi a+\mu n^{4} \alpha a+\beta^{2} n^{2} \xi\right) x+\mu n^{4} \xi k+\mu n^{6} \alpha k-b^{2} n^{4} k=0 .
\end{aligned}
$$

We want to prove the existence of solutions of equation (3.7) as near as required to the complex axis. Thus, it will be sufficient to prove that for any $\epsilon>0$, we can find solutions of equation (3.7) that are on the right side of the line $\Re\{z\}=-\epsilon$. This is equivalent to prove that there exists a solution of the equation

$$
\begin{gathered}
\rho(x-\epsilon)^{5} J a+\rho(x-\epsilon)^{4} J k n^{2}+\left(\mu n^{2} J a+\beta^{2} n^{2} J+\rho \alpha n^{2} a+\rho \xi a+\right. \\
\left.\rho m^{2}\right)(x-\epsilon)^{3}+\left(\mu n^{4} J k+\rho \alpha n^{4} k+\rho \xi k n^{2}\right)(x-\epsilon)^{2}+\left(-2 b n^{2} m \beta+\right. \\
\left.\mu n^{2} m^{2}+\beta^{2} n^{4} \alpha-b^{2} n^{2} a+\mu n^{2} \xi a+\mu n^{4} \alpha a+\beta^{2} n^{2} \xi\right)(x-\epsilon)+ \\
\mu n^{4} \xi k+\mu n^{6} \alpha k-b^{2} n^{4} k=0,
\end{gathered}
$$

with positive real part. To show this fact, the Hurwitz theorem is used; it assesses that a necessary and sufficient condition to guarantee that solutions of the equation

$$
x^{5}+q_{1} x^{4}+q_{2} x^{3}+q_{3} x^{2}+q_{4} x+q_{5}=0,
$$

have negative real part is:

$$
\begin{gathered}
\Lambda_{1}=q_{1}>0, \quad \Lambda_{2}=\operatorname{det}\left(\begin{array}{cc}
q_{1} & 1 \\
q_{3} & q_{2}
\end{array}\right)>0, \quad \Lambda_{3}=\operatorname{det}\left(\begin{array}{ccc}
q_{1} & 1 & 0 \\
q_{3} & q_{2} & q_{1} \\
q_{5} & q_{4} & q_{3}
\end{array}\right)>0, \\
\Lambda_{4}=\operatorname{det}\left(\begin{array}{cccc}
q_{1} & 1 & 0 & 0 \\
q_{3} & q_{2} & q_{1} & 1 \\
q_{5} & q_{4} & q_{3} & q_{2} \\
0 & 0 & q_{5} & q_{4}
\end{array}\right)>0, \text { and } \Lambda_{5}=\operatorname{det}\left(\begin{array}{ccccc}
q_{1} & 1 & 0 & 0 & 0 \\
q_{3} & q_{2} & q_{1} & 1 & 0 \\
q_{5} & q_{4} & q_{3} & q_{2} & q_{1} \\
0 & 0 & q_{5} & q_{4} & q_{3} \\
0 & 0 & 0 & 0 & q_{5}
\end{array}\right)>0 .
\end{gathered}
$$

In our case, we have

$$
\begin{gathered}
q_{1}=\frac{\rho J k n^{2}-5 \rho \epsilon J a}{\rho J a} \\
q_{2}=\frac{\mu n^{2} J a+\beta^{2} n^{2} J+\rho \alpha n^{2} a+\rho \xi a+\rho m^{2}-4 \rho \epsilon J k n^{2}+10 \rho \epsilon^{2} J a}{\rho J a}
\end{gathered}
$$




$$
\begin{gathered}
q_{3}=\frac{-3\left(\mu n^{2} J a+\beta^{2} n^{2} J+\rho \alpha n^{2} a+\rho \xi a+\rho m^{2}\right) \epsilon+\mu n^{4} J k+\rho \alpha n^{4} k}{\rho J a} \\
+\frac{\rho \xi k n^{2}+6 \rho \epsilon^{2} J k n^{2}-10 \rho \epsilon^{3} J a}{\rho J a} \\
q_{4}=\frac{3\left(\mu n^{2} J a+\beta^{2} n^{2} J+\rho \alpha n^{2} a+\rho \xi a+\rho m^{2}\right) \epsilon^{2}+5 \rho \epsilon^{4} J a}{\rho J a} \\
+\frac{-2\left(\mu n^{4} J k+\rho \alpha n^{4} k+\rho \xi k n^{2}\right) \epsilon-4 \rho \epsilon^{3} J k n^{2}-2 b n^{2} m \beta}{\rho J a} \\
+\frac{\mu n^{2} m^{2}+\beta^{2} n^{4} \alpha-b^{2} n^{2} a+\mu n^{2} \xi a+\mu n^{4} \alpha a+\beta^{2} n^{2} \xi}{\rho J a} \\
q_{5}=\frac{-\rho \epsilon^{5} J a-\left(-2 b n^{2} m \beta+\mu n^{2} m^{2}+\beta^{2} n^{4} \alpha-b^{2} n^{2} a+\mu n^{2} \xi a+\mu n^{4} \alpha a+\beta^{2} n^{2} \xi\right) \epsilon}{\rho J a} \\
+\frac{\left(\mu n^{4} J k+\rho \alpha n^{4} k+\rho \xi k n^{2}\right) \epsilon^{2}+\mu n^{4} \xi k+\mu n^{6} \alpha k}{\rho J a} \\
+\frac{-b^{2} n^{4} k+\rho \epsilon^{4} J k n^{2}-\left(\mu n^{2} J a+\beta^{2} n^{2} J+\rho \alpha n^{2} a+\rho \xi a+\rho m^{2}\right) \epsilon^{3}}{\rho J a}
\end{gathered}
$$

We will obtain the existence of $n>1$ such that $\Lambda_{4}<0$.

Lemma 3.1 There exists a polynomial $Q_{0}$ such that

$$
\Lambda_{4}=\frac{Q_{0}\left(\epsilon, n^{10}\right)}{\rho^{3} J^{3} a^{4}}-\frac{2 k^{3} \beta^{2} J(\alpha \rho-J \mu)^{2} n^{12} \epsilon}{\rho^{3} J^{3} a^{4}}+\frac{4 k^{4} \rho J(\rho \alpha-J \mu)^{2} n^{12} \epsilon^{2}}{\rho^{3} J^{3} a^{4}} .
$$

Here $Q_{0}$ is a polynomial in the constitutive coefficients, whose degree is 10 in the parameter $n$ and besides depends on $\epsilon$.

The proof of this lemma is a direct calculation. An appendix is included at the end of the paper where we put forward some sentences in order to obtain $\Lambda_{4}$ with the help of Maple7.

Now, we are able to obtain the main result of this section:

Theorem 3.1 Assume that $\rho \alpha \neq J \mu$ and that $\epsilon$ is sufficiently small. Then, there exists a solution of the problem determined by system (2.14)-(2.16) and initial and boundary data of the form (2.11), (2.12) such that $\Re(\omega)>-\epsilon$.

Proof. The inequality

$$
-\frac{2 k^{3} \beta^{2} J(\rho \alpha-J \mu)^{2}}{\rho^{3} J^{3} a^{4}}+\frac{4 k^{4} \rho J(\rho \alpha-J \mu)^{2} \epsilon}{\rho^{3} J^{3} a^{4}}=m_{\epsilon}<0,
$$

is satisfied for sufficiently small $\epsilon>0$.

By taking $n$ sufficiently large, we see that

$$
\Lambda_{4}=\epsilon m_{\epsilon} n^{12}+\frac{Q_{0}\left(\epsilon, n^{10}\right)}{\rho^{3} J^{3} a^{4}}<0 .
$$


Then, there exists a solution (for $n$ sufficiently large) of the problem such that $\omega$ is on the right side of the line $\Re\{z\}=-\epsilon$.

We can consider the same analysis for boundary conditions of the type:

$$
u_{x}(x, t)=\phi(x, t)=\theta(x, t)=0, \quad x=0, \pi .
$$

whenever we assume that

$$
\int_{0}^{\pi} u^{0}(x) d x=\int_{0}^{\pi} v^{0}(x) d x=0 .
$$

The solutions are combinations of functions of the form

$$
u=A \exp (\omega t) \cos n x, \quad \phi=B \exp (\omega t) \sin n x, \quad \theta=C \exp (\omega t) \sin n x .
$$

The parameter $\omega$ must satisfy equation (3.7) and our analysis can be repeated.

It is also clear that the analysis does not depend on the length of the interval. Thus, our result applies to every interval of finite length.

Now, we give an alternative argument to prove the slow decay. We make the analysis in the particular case that $\rho=J=a=1$. Now, we assume boundary conditions

$$
u(x, t)=\phi(x, t)=\theta(x, t)=0, \quad x=0, \pi,
$$

but the analysis also works for boundary conditions (3.1) or (3.20). In [13] it was proved that solutions are given by means of a semigroup of contractions. In fact for $U=(u, \phi)$, we can write our system in the form

$$
\frac{d^{2} U}{d t^{2}}=-A U+B^{*} \theta, \frac{d \theta}{d t}=-B \frac{d U}{d t}-C \theta
$$

in the Hilbert space $H_{0}^{1} \times H_{0}^{1} \times L^{2}$, where the operators are defined in the following way

$$
\begin{gathered}
A=\left(\begin{array}{cc}
-\mu D^{2} & -b D \\
b D & -\alpha D^{2}+\xi
\end{array}\right), \quad D(A)=\left(H^{2} \cap H_{0}^{1}\right)^{2}, \\
B=\left(\begin{array}{cc}
-\beta D & m
\end{array}\right), \quad D(B)=H_{0}^{1} \times L^{2}, \\
C=-k D^{2}, \quad D(C)=H^{2} \cap H_{0}^{1},
\end{gathered}
$$

and $D=d / d x$. We have that $D\left(C^{1 / 2}\right) \subset D(B), D\left(A^{1 / 2}\right) \subset D\left(B^{*}\right)$ and $A^{1 / 2} B C^{-1}$ is densely defined and bounded from $H_{2}$ to $H_{1}$. In this situation we can apply the result of Henry et al. [8] which proves that our semigroup is exponentially stable if and only if the semigroup generated by the operator

$$
M=\left(\begin{array}{cc}
0 & I \\
-A & -B C^{-1} B^{*}
\end{array}\right),
$$

is exponentially stable. After some calculations and the use of the compact perturbation argument we can reduce the exponential stability of our system to one of the form

$$
\begin{aligned}
& \mu u_{x x}+b \phi_{x}-\beta \dot{u}=\ddot{u} \\
& \alpha \phi_{x x}-b u_{x}-\xi \phi=\ddot{\phi}
\end{aligned}
$$


which is not exponentially stable whenever $\alpha \neq \mu$. One thinks that it is not difficult to extend these arguments to the general case, but we need a suitable extension of the decoupling techniques proposed by Henry et al. [8] to our case.

Likewise it is worth noting that following a similar procedure, these arguments can be applied to alternative boundary conditions. The case $\rho \alpha=J \mu$ deserves a particular analysis as well. In fact the so called results for the Timoshenko beams suggests the exponential stability in this case.

\section{Exponential Stability: Case of microtemperatures}

In this section we consider the problem determined by evolution equations (2.7)-(2.10) with initial conditions (2.11) and boundary conditions (2.12). It is worth recalling that Iesan [9] has proved the existence of solutions for the $n$-dimensional problem. Here, we use the semigroup approach to prove the exponential stability of solutions for the onedimensional problem. Our methodology is based on the arguments proposed in the book of Liu and Zheng [16]. Thus, it will be suitable to remember the semigroup approach to this problem. To make the calculations easier we assume boundary conditions

$$
u=w=\theta_{x}=\phi_{x}=0, \text { for } x=0, \pi .
$$

The other boundary conditions proposed in (2.12) could be analysed in a similar way, but they could need the Gagliardo-Nirenberg inequality. Nevertheless, the basic analysis would be the same.

For $\omega=(u, v, \phi, \varphi, \theta, w)$ we consider the Hilbert space

$$
\mathcal{H}=\left\{\omega \in H_{0}^{1} \times L^{2} \times H^{1} \times L^{2} \times L^{2} \times L^{2}, \int_{0}^{\pi} \phi d x=\int_{0}^{\pi} \varphi d x=\int_{0}^{\pi} \theta d x=0\right\} .
$$

It is worth recalling that in this situation the inner product can be given as

$$
\begin{gathered}
<(u, v, \phi, \varphi, \theta, w),\left(u^{*}, v^{*}, \phi^{*}, \varphi^{*}, \theta^{*}, w^{*}\right)>_{\mathcal{H}} \\
=\int_{0}^{\pi}\left(\rho v \bar{v}^{*}+J \varphi \bar{\varphi}^{*}+c \theta \bar{\theta}^{*}+\delta w \bar{w}^{*}+\mu u_{x} \bar{u}_{x}^{*}+\alpha \phi_{x} \bar{\phi}_{x}^{*}+\xi \phi \bar{\phi}^{*}+b\left(u_{x} \bar{\phi}^{*}+\bar{u}_{x}^{*} \phi\right)\right) d x
\end{gathered}
$$

The solutions of this problem are defined by the semigroup of contractions generated by the operator

$$
\mathcal{A}=\left(\begin{array}{cccccc}
0 & I d & 0 & 0 & 0 & 0 \\
\rho^{-1} \mu D^{2} & 0 & \rho^{-1} b D & 0 & -\rho^{-1} \beta D & 0 \\
0 & 0 & 0 & I d & 0 & 0 \\
-J^{-1} b D & 0 & J^{-1}\left(\alpha D^{2}-\xi\right) & 0 & J^{-1} m & -J^{-1} d D \\
0 & -c^{-1} \gamma D & 0 & -c^{-1} l & c^{-1} k D^{2} & c^{-1} \kappa_{1} D \\
0 & 0 & 0 & -\delta^{-1} d D & -\delta^{-1} \kappa_{3} D & \delta^{-1}\left(\kappa_{4} D^{2}-\kappa_{2}\right)
\end{array}\right) .
$$


To prove the exponential stability we show that

$\{i \lambda, \lambda$ is real $\}$ is contained in the resolvent of $\mathcal{A}$,

and that

$$
\varlimsup_{|\lambda| \rightarrow \infty}||(i \lambda \mathcal{I D}-\mathcal{A})^{-1}||<\infty .
$$

First, we prove that 0 is in the resolvent of the operator $\mathcal{A}$.

Lemma 4.1 Let $\mathcal{A}$ defined in (4.3). Then 0 is in the resolvent of $\mathcal{A}$.

Proof. For any $\mathcal{F}=\left(f_{1}, f_{2}, f_{3}, f_{4}, f_{5}, f_{6}\right) \in \mathcal{H}$, we want to find $\omega \in \mathcal{H}$ such that

$$
\mathcal{A} \omega=\mathcal{F}
$$

i. e.

$$
\begin{gathered}
v=f_{1}, \\
\rho^{-1} \mu D^{2} u+\rho^{-1} b D \phi-\rho^{-1} \beta D \theta=f_{2}, \\
\varphi=f_{3}, \\
-J^{-1} b D u+J^{-1}\left(\alpha D^{2}-\xi\right) \phi+J^{-1} m \theta-J^{-1} d D w=f_{4}, \\
-c^{-1} \gamma D v-c^{-1} l \varphi+c^{-1} k D^{2} \theta+c^{-1} \kappa_{1} D w=f_{5}, \\
-\delta^{-1} d D \varphi-\delta^{-1} \kappa_{3} D \theta+\delta^{-1}\left(\kappa_{4} D^{2}-\kappa_{2}\right) w=f_{6} .
\end{gathered}
$$

From (4.11) and (4.12) we can write

$$
\begin{aligned}
& c^{-1} k D^{2} \theta+c^{-1} \kappa_{1} D w=f_{5}+c^{-1} l f_{3}+c^{-1} \gamma D f_{1} \in L^{2} . \\
& -\delta^{-1} \kappa_{3} D \theta+\delta^{-1}\left(\kappa_{4} D^{2}-\kappa_{2}\right) w=f_{6}+\delta^{-1} d D f_{3} \in L^{2} .
\end{aligned}
$$

In view of the constitutive assumptions and the regularity theory of linear elliptic operators we obtain the existence of solutions $\theta, w \in H^{2}$. Thus, $\theta, w$ as the solution of (4.13) and (4.14) are substituted in (4.8) and (4.10) to get

$$
\begin{gathered}
\rho^{-1} \mu D^{2} u+\rho^{-1} b D \phi=f_{2}+\rho^{-1} \beta D \theta \\
-J^{-1} b D u+J^{-1}\left(\alpha D^{2}-\xi\right) \phi=f_{4}-J^{-1} m \theta+J^{-1} d D w
\end{gathered}
$$

Consequently the unique solvability follows. It is clear from the regularity theory of linear elliptic equations that

$$
\|\omega\|_{\mathcal{H}} \leq K\|\mathcal{F}\|_{\mathcal{H}}
$$

where $K$ is a constant independent of $\omega$.

Now, we are in situation to prove (4.4).

Lemma 4.2 Let $\mathcal{A}$ defined in (4.3). Then condition (4.4) holds. 
Proof. The proof consists of the following steps:

(i) Using the contraction mapping theorem and since 0 is in the resolvent of $\mathcal{A}$, for any real $\lambda$ such that $|\lambda|<\left\|\mathcal{A}^{-1}\right\|^{-1}$, the operator $i \lambda \mathcal{I D}-\mathcal{A}=\mathcal{A}\left(i \lambda \mathcal{A}^{-1}-\mathcal{I D}\right)$ is invertible. Moreover, $\left\|(i \lambda \mathcal{I D}-\mathcal{A})^{-1}\right\|$ is a continuous function of $\lambda$ in the interval $\left(-\left\|\mathcal{A}^{-1}\right\|^{-1},\left\|\mathcal{A}^{-1}\right\|^{-1}\right)$.

(ii) If $\sup \left\{\left\|(i \lambda \mathcal{I D}-\mathcal{A})^{-1}||,|\lambda|<\right\| \mathcal{A}^{-1} \|^{-1}\right\}=M<\infty$, then by the contraction theorem, the operator

$$
i \lambda \mathcal{I D}-\mathcal{A}=\left(i \lambda_{0} \mathcal{I D}-\mathcal{A}\right)\left(\mathcal{I D}+i\left(\lambda-\lambda_{0}\right)\left(i \lambda_{0} \mathcal{I D}-\mathcal{A}\right)^{-1}\right)
$$

is invertible for $\left|\lambda-\lambda_{0}\right|<M^{-1}$. By choosing $\lambda_{0}$ as close to $\left\|\mathcal{A}^{-1}\right\|^{-1}$ as possible, we conclude that the set $\left\{\lambda,|\lambda|<\left\|\mathcal{A}^{-1}\right\|^{-1}+M^{-1}\right\}$ is contained in the resolvent of $\mathcal{A}$ and $\|(i \lambda \mathcal{I D}-\mathcal{A})^{-1}||$ is a continuous function of $\lambda$ in the interval $\left(-\left\|\mathcal{A}^{-1}\right\|^{-1}-M^{-1},\left\|\mathcal{A}^{-1}\right\|^{-1}+\right.$ $\left.M^{-1}\right)$.

(iii) If (4.4) were not true, then there would exist a real number $\varpi$ with $\left\|\mathcal{A}^{-1}\right\|^{-1} \leq$ $|\varpi|<\infty$ such that the set $\{i \lambda,|\lambda|<|\varpi|\}$ would be in the resolvent of $\mathcal{A}$ and $\sup \{\|(i \lambda \mathcal{I} \mathcal{D}-$ $\left.\mathcal{A})^{-1}||,|\lambda|<|\varpi|\right\}=\infty$. As a result there would exist a sequence of real numbers $\lambda_{n}$ with $\lambda_{n} \rightarrow \varpi,\left|\lambda_{n}\right|<|\varpi|$ and a sequence of unit norm vectors $\omega_{n}=\left(u_{n}, v_{n}, \phi_{n}, \varphi_{n}, \theta_{n}, w_{n}\right)$ in the domain of the operator such that

$$
\left\|\left(i \lambda_{n} \mathcal{I D}-\mathcal{A}\right) \omega_{n}\right\| \rightarrow 0
$$

That is to say

$$
\begin{gathered}
i \lambda_{n} u_{n}-v_{n} \rightarrow 0 \text { in } H_{0}^{1}, \\
i \lambda_{n} v_{n}-\rho^{-1} \mu D^{2} u_{n}-\rho^{-1} b D \phi_{n}+\rho^{-1} \beta D \theta_{n} \rightarrow 0 \text { in } L^{2}, \\
i \lambda_{n} \phi_{n}-\varphi_{n} \rightarrow 0 \text { in } H_{0}^{1}, \\
i \lambda_{n} \varphi_{n}+J^{-1} b D u_{n}-J^{-1}\left(\alpha D^{2}-\xi\right) \phi_{n}-J^{-1} m \theta_{n}+J^{-1} d D w_{n} \rightarrow 0 \text { in } L^{2}, \\
i \lambda_{n} \theta_{n}+c^{-1} \gamma D v_{n}+c^{-1} l \varphi_{n}-c^{-1} k D^{2} \theta_{n}-c^{-1} \kappa_{1} D w_{n} \rightarrow 0 \text { in } L^{2}, \\
i \lambda_{n} w_{n}+\delta^{-1} d D \varphi_{n}+\delta^{-1} \kappa_{3} D \theta_{n}-\delta^{-1}\left(\kappa_{4} D^{2} w_{n}-\kappa_{2} w_{n}\right) \rightarrow 0 \text { in } L^{2} .
\end{gathered}
$$

Taking the inner product of $\left(i \lambda_{n} \mathcal{I D}-\mathcal{A}\right) \omega_{n}$ with $\omega_{n}$ in $\mathcal{H}$, its real part yields

$$
\left\|D \theta_{n}\right\|,\left\|D w_{n}\right\| \rightarrow 0
$$

Using the Poincaré inequality and (4.1) we find that $w_{n} \rightarrow 0$. From (4.24), we see that

$$
\lambda_{n}^{-1}\left[\delta^{-1} d D \varphi_{n}-\delta^{-1} \kappa_{4} D^{2} w_{n}\right] \rightarrow 0 \text { in } L^{2} .
$$

Dividing (4.21) by $\lambda_{n}$, we obtain

$$
\delta^{-1} d i D \phi_{n}-\lambda_{n}^{-1} \delta^{-1} \kappa_{4} D^{2} w_{n} \rightarrow 0 \text { in } L^{2}
$$

Since $\left\|D \phi_{n}\right\| \leq 1$, we deduce that $\lambda_{n}^{-1}|| D^{2} w_{n} \|$ is bounded. Taking the inner product of (4.27) by $D \phi_{n}$, we get

$$
\delta^{-1} d i\left\|D \phi_{n}\right\|^{2}+\lambda_{n}^{-1} \delta^{-1} \kappa_{4}\left(D w_{n}, D^{2} \phi_{n}\right) \rightarrow 0
$$


In view of (4.22), $\lambda_{n}^{-1}\left\|D^{2} \phi_{n}\right\|$ is bounded. It follows that

$$
\left\|D \phi_{n}\right\| \rightarrow 0
$$

In a similar way from (4.23), we have

$$
\lambda_{n}^{-1}\left[c^{-1} \gamma D v_{n}-c^{-1} k D^{2} \theta_{n}\right] \rightarrow 0 \text { in } L^{2} .
$$

Dividing (4.19) by $\lambda_{n}$, we obtain

$$
c^{-1} \gamma i D u_{n}-\lambda_{n}^{-1} c^{-1} k D^{2} \theta_{n} \rightarrow 0 \text { in } L^{2} .
$$

Since $\left\|D u_{n}\right\| \leq 1$, it turns out that $\left\|\lambda_{n}^{-1} D^{2} \theta_{n}\right\|$ is bounded. Taking the inner product of (4.31) by $D u_{n}$, we find

$$
c^{-1} \gamma i\left\|D u_{n}\right\|^{2}+\lambda_{n}^{-1} c^{-1} k\left(D \theta_{n}, D^{2} u_{n}\right) \rightarrow 0 .
$$

In view of (4.20) $\lambda_{n}^{-1}\left\|D^{2} u_{n}\right\|$ is bounded. It follows that

$$
\left\|D u_{n}\right\| \rightarrow 0
$$

Now from (4.19), (4.33) and (4.21), (4.29), we also have

$$
\lambda_{n}^{-1} D v_{n}, \lambda_{n}^{-1} D \varphi_{n}, \rightarrow 0 \text { in } L^{2} .
$$

Thus, from (4.20) and (4.22) we obtain

$$
\begin{aligned}
i\left\|v_{n}\right\|^{2}+\rho^{-1} \mu\left(D u_{n}, \lambda_{n}^{-1} D v_{n}\right) & \rightarrow 0, \\
i\left\|\varphi_{n}\right\|^{2}+J^{-1} \alpha\left(D \phi_{n}, \lambda_{n}^{-1} D \varphi_{n}\right) & \rightarrow 0 .
\end{aligned}
$$

Therefore, we arrive at

$$
v_{n}, \varphi_{n} \rightarrow 0 \text { in } L^{2}
$$

The conditions (4.25), (4.29), (4.33), (4.37) contradict that the sequence has unit norm. Then condition (4.4) is also satisfied.

Lemma 4.3 Let $\mathcal{A}$ be the operator defined in (4.3). Then condition (4.5) holds.

Proof. We now prove (4.5) by a contradiction argument. Suppose that (4.5) does not hold. Then, there exists a sequence $\lambda_{n}$ with $\left|\lambda_{n}\right| \rightarrow \infty$ and a sequence of complex vectors $\omega_{n}=\left(u_{n}, v_{n}, \phi_{n}, \varphi_{n}, \theta_{n}, w_{n}\right)$ with unit norm and in the domain of the operator such that (4.18) holds. Now, the proof is similar to the one in lemma 4.2, because the only argument we need is that $\lambda_{n}$ does not tend to zero.

We have proved:

Theorem 4.1 Let $(u, \phi, \theta, w)$ be a solution of the thermoelastic problem determined by system (2.7)-(2.10) with boundary conditions (4.1) and initial conditions (2.11). Then the solution decay exponentially. That is, there exist two positive constants $M, \Omega$ such that

$$
\|(u(t), \phi(t), \theta(t), w(t))\| \leq M \exp (-\Omega t)\|(u(0), \phi(0), \theta(0), w(0))\| .
$$

Proof. As (4.4) and (4.5) hold we can conclude the exponential decay of the solutions (see $[16,23])$. 


\section{Exponential Stability: Heat conduction problem}

In this section we concentrate our attention in the three-dimensional heat conduction problem. In this situation the system of equations is

$$
\begin{gathered}
k \Delta \theta+\kappa_{1} \operatorname{div} \mathbf{w}=c \dot{\theta} \\
\kappa_{6} \triangle \mathbf{w}+\left(\kappa_{4}+\kappa_{5}\right) \operatorname{grad} \operatorname{div} \mathbf{w}-\kappa_{3} \operatorname{grad} \theta-\kappa_{2} \mathbf{w}=\delta \dot{\mathbf{w}}
\end{gathered}
$$

where $c=a T_{0}$.

We assume that this system is satisfied in a domain $B=[0, L] \times \Sigma$, where $\Sigma$ is a bounded domain in the 2-dimensional Euclidean space and $L$ is a positive constant. Initial and boundary conditions are determined by

$$
\theta(\mathbf{x}, 0)=\theta^{0}, \quad \mathbf{w}(\mathbf{x}, 0)=\mathbf{w}^{0} \text { in } B
$$

and

$$
\theta(\mathbf{x}, t)=0, \mathbf{w}(\mathbf{x}, t)=\mathbf{0} \text { on } \partial B
$$

respectively.

In [13] it was pointed out that if we define the function

$$
E_{h}(t)=\frac{1}{2} \int_{B}\left(c \theta^{2}+\delta \mathbf{w} \cdot \mathbf{w}\right) d v
$$

we have

$$
E_{h}(t) \leq E_{h}(0) \exp \left(-\delta^{*} t\right)
$$

where $\delta^{*}$ is a calculable positive constant. This is a result on exponential stability in the 3-dimensional heat conduction problem. It is worth noting that we can use a similar argument in the case of other kind of boundary conditions.

A particular class of solutions of the heat conduction problem is the anti-plane thermal deformations. The functions of the form:

$$
\theta=\theta\left(x_{2}, x_{3}, t\right), \quad w_{2}=w_{3}=0, \quad w_{1}=w_{1}\left(x_{2}, x_{3}, t\right)
$$

are solutions of system (5.1),(5.2) whenever $\theta$ and $w_{1}$ satisfy the equations

$$
c \dot{\theta}=k \theta_{, \alpha \alpha}, \quad \delta \dot{w}_{1}=\kappa_{6} w_{1, \alpha \alpha}-\kappa_{2} w_{1}
$$

where $\alpha=2,3$. This system is not coupled and we can obtain the rate of decay easily.

The solutions of equation (5.8) 1 are of the form

$$
\exp \left(-\frac{\lambda_{n} k}{c} t\right) \Phi_{n}\left(x_{2}, x_{3}\right)
$$

where $\Phi_{n}$ satisfies the problem

$$
\Phi_{n, \alpha \alpha}+\lambda_{n} \Phi_{n}=0, \text { in } \Sigma, \quad \Phi_{n}=0 \text {, on } \partial \Sigma .
$$

Thus the rate of decay for $\theta$ is of the type $\exp \left(-\frac{\lambda_{1} k}{c} t\right)$.

The solutions of the equation $(5.8)_{2}$ are of the form

$$
\exp \left(-\left(\frac{\lambda_{n} \kappa_{6}}{\delta}+\frac{\kappa_{2}}{\delta}\right) t\right) \Phi_{n}\left(x_{2}, x_{3}\right)
$$

Thus the rate of decay for $w_{1}$ is of the type $\exp \left(-\left(\frac{\lambda_{1} \kappa_{6}}{\delta}+\frac{\kappa_{2}}{\delta}\right) t\right)$. 


\section{References}

[1] M. Ciarletta and D. Iesan, Non-classical elastic solids. Pitman Research Notes in mathematics Series 293. New York (1993).

[2] C. M. Dafermos, Contraction semigroups and trend to equilibrium in continuum mechanics, in P. Germain and B. Nayroles (Eds.) Applications of Methods of Functional Analysis to Problems in Mechanics, Springer Lecture Notes in Math. 507, Springer, Berlin, 1976, pp. 295-306.

[3] R. Grot, Thermodynamics of continuum with microstructure. Int. Jour. Engn. Sci., 7, 801-814, (1969).

[4] A. C. Eringen, Mechanics of micromorphic materials, in: H.Gortler (Ed.), Proc. 11th. Congress of Appl. Mech., Springer, New York, 1964

[5] A. C. Eringen, Mechanics of micromorphic continua, in: E.Kroner (Ed.), Mechanics of Generalized Continua, Springer, Berlin, 1967, pp. 18-35.

[6] A. C. Eringen, C. B. Kafadar, Polar field theories, in: A.C. Eringen (Ed.), Continuum Physics vol. IV, Academic Press, New York, 1976

[7] A. C. Eringen, Microcontinuum Field Theories, Springer, Berlin, 1999.

[8] D.B. Henry, A. Perissinito and O. Lopes, On the essential spectrum of a semigroup of thermoelasticity. Nonlinear Analysis, TMA 21, (1993) 67-75.

[9] D. Iesan, On a theory of micromorphic elastic solids with microtemperatures. Jour. Thermal Stresses, 24, 737-752, (2001).

[10] D. Iesan, On the micromorphic thermoelasticity. Int. Jour. Engn. Sci., 40, 549-567, (2002).

[11] D. Iesan, On the theory of heat conduction in micromorphic continua. Int. Jour. Engn. Sci., 40, 1859-1878, (2002).

[12] D. Iesan and L. Nappa, Extremum principles and existence results in micromorphic elasticity. Int. Jour. Engn. Sci., 39, 2051-2070, (2001).

[13] D. Iesan and R. Quintanilla, On a theory of thermoelasticity with microtemperatures. Jour. Thermal Stresses, 23, 199-215, (2000).

[14] S. Jiang and R. Racke, Evolution Equations in Thermoelasticity, Chapman and Hall/CRC, Boca Raton, 2000.

[15] G. Lebeau and E. Zuazua, Decay rates for the three-dimensional linear systems of thermoelasticity. Arch. Rat. Mech. Anal., 148, 179-231, (1999).

[16] Z. Liu and S. Zheng, Semigroups associated with dissipative systems Chapman and Hall/CRC, Boca Raton, 1999. 
[17] R. Quintanilla and R. Racke, Stability for thermoelasticity of type III. Discrete and Continuous Dynamical Systems, B, 3, 383-400, (2003).

[18] P. Riha, On the theory of heat conducting micropolar fluids with microtemperatures. Acta Mech., 23, 1-8, (1975).

[19] P. Riha, On the microcontinuum model of heat conduction in materials with inner structure. Int.Jour. Engn. Sci., 14, 529-535, (1976).

[20] P. Riha, Poiseuille flow of microthermopolar fluids. Acta Technica CSAV , 22, 602614, (1977).

[21] R. Racke, Thermoelasticity with second sound- exponential stability in linear and non-linear 1-d. Math. Meth.Appl. Sci., 25, 409-441, (2002).

[22] M. Slemrod, Global existence, uniqueness and asymptotic stability of classical smooth solutions in one-dimensional nonlinear thermoelasticity Arch. Rational Mech. Anal. 76, (1981) 97-133.

[23] A. Wyler, Stability of wave equation with dissipative boundary conditions in a bounded domain. Differential and Integral Equations 7, (1994), 345-366.

[24] X.Zhang and E. Zuazua, Decay of solutions of the system of thermoelasticity of type III. Communications in Contemporary Mathematics, 5, 25-83 (2003).

\section{APPENDIX}

In order to carry out the calculations presented in this paper, we have used the program Maple7. The sequence of "sentences" we have needed in section 2 is:

$$
>\text { with(Linear Algebra): }
$$

$$
\begin{gathered}
Z:=<<r h o * x^{2}+m u * n^{2}, b * n, \text { beta } * x * n>\mid<b * n, J * x^{2}+\text { alpha } * n^{2}+x i, m * x> \\
\mid<- \text { beta } * n,-m, a * x+k * n^{2}>>; \\
\text { Determinant }(Z) ; \\
\text { collect }(\%, x) ; \\
\text { subs }(x=x-\text { epsilon, } \%) ; \\
\text { collect }(\%, x) ; \\
q_{1}:=\left(\text { rho } * J * k * n^{2}-5 * \text { rho } * \text { epsilon } * J * a\right) /(\text { rho } * J * a) ;
\end{gathered}
$$




$$
q_{2}:=\left(m u * n^{2} * J * a+\operatorname{beta}^{2} * n^{2} * J+r h o * a l p h a * n^{2} * a+r h o * x i * a\right.
$$

$+r h o * m^{2}-4 * r h o *$ epsilon $* J * k * n^{2}+10 * r h o *$ epsilon $\left.^{2} * J * a\right) /($ rho $* J * a)$;

$$
\begin{aligned}
& q_{3}:=(-3 *\left(m u * n^{2} * J * a+\text { beta }^{2} * n^{2} * J+r h o * a l p h a * n^{2} * a+r h o * x i * a+r h o * m^{2}\right) * \text { epsilon } \\
&+ m u * n^{4} * J * k+r h o * \text { alpha } * n^{4} * k+r h o * x i * k * n^{2} \\
&\left.+6 * r h o * \text { epsilon }{ }^{2} * J * k * n^{2}-10 * r h o * \text { epsilon }^{3} * J * a\right) /(r h o * J * a)
\end{aligned}
$$

$q_{4}:=\left(3 *\left(m u * n^{2} * J * a+\right.\right.$ beta $\left.^{2} * n^{2} * J+r h o * a l p h a * n^{2} * a+r h o * x i * a+r h o * m^{2}\right) * e p s i l o n^{2}$ $+5 * r h o * e_{p s i l o n}^{4} * J * a-2 *\left(m u * n^{4} * J * k+r h o * a l p h a * n^{4} * k+r h o * x i * k * n^{2}\right) *$ epsilon $-4 * r h o *$ epsilon $^{3} * J * k * n^{2}-2 * b * n^{2} * m *$ beta $+m u * n^{2} * m^{2}+$ beta $^{2} * n^{4} *$ alpha $-b^{2} * n^{2} * a$ $\left.+m u * n^{2} * x i * a+m u * n^{4} * a l p h a * a+b^{2} a^{2} * n^{2} * x i\right) /(r h o * J * a) ;$

$q_{5}:=\left(-\right.$ rho $*$ epsilon ${ }^{5} * J * a-\left(-2 * b * n^{2} * m *\right.$ beta $+m u * n^{2} * m^{2}+$ beta $a^{2} * n^{4} * a l p h a-b^{2} * n^{2} * a$ $+m u * n^{2} * x i * a+m u * n^{4} *$ alpha $* a+$ beta $\left.^{2} * n^{2} * x i\right) *$ epsilon $+\left(m u * n^{4} * J * k+r h o *\right.$ alpha $\left.* n^{4} * k+r h o * x i * k * n^{2}\right) * e_{\text {epsilon }}^{2}+m u * n^{4} * x i * k$ $+m u * n^{6} *$ alpha $* k-b^{2} * n^{4} * k+$ rho $*$ epsilon $^{4} * J * k * n^{2}$

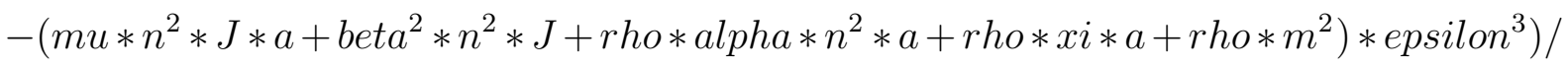
$(r h o * J * a)$ $M 4:=<<q_{1}, q_{3}, q_{5}, 0>\left|<1, q_{2}, q_{4}, 0>\right|<0, q_{1}, q_{3}, q_{5}>\mid<0,1, q_{2}, q_{4}>>$;

$$
d 4:=\operatorname{Determinant}(M 4)
$$

collect(d4, epsilon);

$\operatorname{collect}(d 4, n)$ 